\title{
Lessons for Successful Cognitive Developmental Science in Educational Settings: The Case of Executive Functions
}

\author{
Michelle R. Ellefson \\ Sara T. Baker \\ Jenny L. Gibson \\ University of Cambridge Faculty of Education
}

This manuscript was accepted for publication 15th November 2018 by Journal of Cognition and Development. This is the version submitted on $9^{\text {th }}$ November 2018 (it was the second revision).

DOI: https://doi.org/10.1080/15248372.2018.1551219

\section{Author Notes}

Correspondence concerning this article should be addressed to Michelle R. Ellefson, University of Cambridge Faculty of Education, 184 Hills Road, Cambridge, CB2 8PQ, United Kingdom. Email: mre33@cam.ac.uk. Ideas presented in this paper were supported by the LEGO Foundation, Economic and Social Research Council (ES/K010255/1, ES/N006577/1, ES/N009266/1), Arts and Humanities Research Council (AH/N004671/1) and the Institute of Education Sciences, U.S. Department of Education (R305A110932) through grants to the University of Cambridge. The opinions expressed are those of the authors and do not represent views of the Institute or the U.S. Department of Education. Thanks to our (1) educator colleagues from the Spinney Primary School, (2) some of our own graduate students, who are also professional educators, for thoughtful discussions of the issues raised in the present paper, and (3) Ric Parkin for proofreading. 


\begin{abstract}
This paper gives a reflective account of lessons learned from the experiences of three cognitive developmental scientists conducting psychological research in educational settings. First, we describe and analyze four key cultural distinctions between traditional approaches to research in psychology and education: (1) different structures; (2) different research philosophies; (3) different time frames; and (4) different semantics. Valuing and understanding the rationale behind the differing views from education is vital for creating effective collaborations. Second, we describe how our independent research questions and methods in the area of executive functions have been impacted by classroom constraints and observations, discussions of findings, and planning interventions. We advocate for shared goal setting with educators because it improves the research design, validity generalizability and the impact of findings. We anticipate that as psychologists engage in more transparent research the opportunity to collaborate with educators on educational policy will increase and that these lessons will remain important as we consider policy-making in future research studies.
\end{abstract}




\section{Lessons for Successful Cognitive Developmental Science in Educational Settings: The Case of Executive Functions}

We are three cognitive developmental scientists who work day-to-day with a variety of educational researchers within academic settings as well as educators and educational stakeholders in our own independent streams of research and outreach (including teachers, teacher trainers, school leadership teams, policy makers, educational consultants, educational charity staff, etc.). We are classically trained in experimental psychology and we use traditional experimental and quasi-experimental methods in academic research. In this article we share our independent experiences of successful collaboration with researchers and practitioners from the field of education as well as insight that we have gained from additional conversations with educators from the UK and the USA. It is our intention to provide practical examples of how we addressed some of the challenges of this interdisciplinary working and the benefits that such collaborations can bring. Our intended audience is psychologists who have an interest in applications of cognitive developmental science to educational settings, but who may be unsure of where to begin.

Collectively, our initial training and subsequent work in psychology has been informed by cognitive science, developmental psychology, psycholinguistics and neuroscience. To avoid interchanging these and related terms, we use just one term in this article - psychology. For additional simplicity we refer to those who use experimental or quasi-experimental methods in their psychological research as psychologists. We have focused our reflections on the type of research that psychologists might conduct with educators, while appreciating that some of our experiences might generalize outside of this frame of reference.

The paper is split into two main sections: What Have We Learned? and How Have We Changed? The first part of our paper - What Have We Learned? - explores some of the most important lessons we have learned collectively about educational cultures and how those 
lessons have informed our current practices. We outline the nature of such differences and give examples of practices that facilitate bidirectional working with educational researchers and teachers. After starting jobs in our Faculty of Education, we learned that what we had once considered to be diverse areas of psychological research all fell into only one of many different research philosophies found in education. Understanding how our toolbox of psychological methods was viewed as only a small part of the scope of educational research was one key lesson for us to understand as we started conducting studies in education. Those early conversations with our colleagues and students helped us become aware of the structural and cultural differences between psychological and educational research. For example, when talking to an educational audience rather than a psychological one, we had to develop responses to questions like: "Is it meaningful to reduce children to numbers?", which people posed when we described our statistical findings. The lessons we have chosen to highlight in this section include differences in structures, research philosophies, time frames and semantics.

The second part of our paper - How Have We Changed? - showcases our individual research programs and how they have evolved since we started working with education. As our understanding of key cultural differences improved, so too did our ability to reach out to educators outside of our academic networks. Importantly, we have discovered that our willingness to learn and engage with educational cultures has paid remarkable dividends in improving the validity of our own theories and data. In recent years, our shared goal setting has extended to include educational policy makers. Policy decisions for education are multifaceted and we are learning that research evidence is just one part of the puzzle. Psychology findings can be much more influential if they account for this complexity from its earliest conception through shared agenda setting and the co-creation of more appropriate research designs that benefit both education and psychology. We conclude the paper with some reflections on how our collaborations with educators has prepared us to think more about joining with them to 
influence educational policy and how our research will continue to change as we build bridges between research and policy.

\section{What Have We Learned? \\ Context and Culture Matter}

Considering the broader context of our research is essential for generating knowledge that is relevant for educators. Many psychologists work in academic institutions with an education department (or school, college or faculty, depending on institutional labels). These departments may seem the natural first port of call for collaboration or cross-fertilization of ideas. However, there are differences of approach between academics based in education and those based in psychology that need to be considered carefully when attempting collaborative work. An important lesson we have learned is to gain some orientation to the various landscapes of education. In this section we discuss examples from our work in academia, as well as working with schools and local and national government. We have experience working with schools in the UK and USA; we use those specific contexts as examples, but we acknowledge that there are many other structures beyond these two countries. We focus on four main areas of challenge that we have experienced in building bridges between psychology and education, and the lessons we have drawn in working through them: (1) different structures; (2) different research philosophies; (3) different time frames; and (4) different semantics. One of the key benefits of having experienced these challenges is that it made us reflect more deeply about our scholarship and it has changed the way we plan, carry out, and communicate our findings. By sharing some reflections here, we hope that other psychologists can benefit from some lessons we have learned.

\section{Lesson I: Different Structures}

There are divergent views regarding the status of education as an academic discipline. An influential view is that education is not an academic discipline in its own right, but a broader field 
of enquiry to which academic disciplines such as psychology, history, philosophy, economics, linguistics and sociology all contribute (McCulloch, 2002). McCulloch describes the tension arising from these various disciplines within education when following methods of their "parent discipline', whilst also orienting to an educationalist perspective. Furthermore, he notes that much of the foundational academic enquiry that has flourished in education departments has been based in the humanities, rather than empirical sciences.

Organization and resources vary across different academic departments. The practical implication of these structural observations is that as psychologists seeking collaborations with academic colleagues based in education, we have learned that we should not assume a shared set of expectations about how and why the research project is conducted. Some of the academic practices, as well as the organizational and physical structures that we, as psychologists, take for granted do not tend to exist in most educational departments. In our department, tightly structured groups working on related topics and led by a senior academic (i.e., labs) are not common; observation or laboratory testing space is minimal; and collaborative publication (multiple-authored papers) with students is rare. Colleagues in our department have been open to us developing these kinds of structures and working practices, and we have ongoing discussions with our graduate students and postdocs about what it means to work across disciplines. We see this milieu as a strength, in that it forces us to explicitly question and justify our ways of working and decisions about theory and research design.

\section{Institutional structures in education outside of academia are complex and}

changing. In addition to the structural constraints in academic settings, there are a wide variety of structures that exist in educational communities in wider society (e.g., school boards, local education authorities, teaching school alliances, academy chains, charter schools, etc.). One key structural factor, in addition to the macrocosm that each school belongs to, comprises individual school-level policies and leadership. It is vital for psychologists to be able to explain how their findings are valuable and align with the priorities of individual schools who partner in 
their studies. Structural factors have encouraged us to clearly convey how the school can contribute to psychology and how we, as psychologists, can contribute to the school. We have seen tight budgets and limited staff time make school leaders reluctant to embark on even small research projects unless they see a direct benefit to the school. In our work, we allow time and financial resources to give back to the schools. In one example, we co-planned school-wide events with the teaching staff about brain development.

Another structural factor that has affected our research projects is turnover in school leaders and educators without much warning. Projects that had the full support of one educator might not be supported by the next. As a result, long-term projects have substantial risks that have to be integrated into grants and graduate student project planning. Teachers have expressed to us their own concerns about this issue. As a result, we have found that having diverse stakeholder representation on research advisory boards can help to mitigate these risks (see Table 1). For example, in a recent project where a school withdrew from the study due to personnel change, an advisory board member supported us to find a new school to participate.

Sometimes government policies for education can have an impact on what or how studies are conducted. For example, one consequence of the No Child Left Behind policy in the USA was the increased pressure for each student to perform well during annual testing (on a smaller scale, similar high stakes testing occurs in the UK). We have found that schools are now less willing for their students to be taken out of a lesson to participate in an experiment. We find that it can be difficult to conduct studies in the few months leading up to, and during, the testing season. We have learned to either modify our research questions to avoid the ages that are included in this testing or to only collect data from those ages at certain times of the school year. We have found it useful to ask schools about any pressure points when data collection should be avoided in the course of the school year, for example if there is a scheduled inspection or audit. 
School level policies and leadership are often embedded within regional structures (e.g., local educational authorities or academy trusts in the UK or school boards and state-wide structures in the USA). Schools can be influenced by national policies, but there are also instances in the UK and USA where policies are decentralized, and schools have more autonomy. Further distinctions and structures exist between private fee-paying schools and taxpayer-funded schools. Each of these structural layers adds complexity. Psychologists might engage with and learn about these complexities as their research requires. For example, to run one study in the USA, we had to obtain ethical approval from the university's own Institutional Review Board, as well as obtaining approval from the local school board. Both of these bodies required detailed applications. Both stipulated that they would not provide approval until the other had done so, placing the team in a stalemate and requiring delicate negotiations. We have learned that sometimes ethical approval can be complicated when conducting data collection in schools and that policies can vary from school to school. As such, we now ensure that our project planning includes sufficient time when setting up school-based projects. We have learned that different schools have different policies about background checks and whether a psychologist needs a new one to work with that school when they might already have already completed a check through their academic institution. When schools ask for this additional check, it can delay timelines and have serious consequences for grants or graduate student progress.

\section{Lesson II: Different Research Philosophies}

Our second area of learning has been that the importance of the experimental method found in psychology is, at times, criticized in educator circles. Although schools in the UK can be very data-driven and school leadership teams in particular are well-versed in quantitative indicators, some educators still object to the idea of 'reducing students to numbers', instead placing the utmost value on the uniqueness of individuals. This view is shared by some educators in other contexts outside of the UK. 
Not everyone has the same ideas about what is good evidence. Before arriving in our posts, we had not heard of the term post-positivism. We learned that post-positivism is one of many philosophies represented in educational research and the wider social sciences and there is great debate about its use in educational settings (e.g., Denzin \& Lincoln, 2017). Psychology almost exclusively resides under the post-positivist umbrella, especially areas that investigate cognition. As such, post-positivism was axiomatic in our own training. Upon joining the Faculty of Education, we had to learn to situate our work in relation to other philosophies.

Positivism from a psychological perspective is the notion that human behaviour can be understood via measurable data (e.g., Coolican, 2014). Post-positivism is the idea that experimental methods reduce the bias of the researcher (e.g., Cohen, Manion \& Morrison, 2011). From this perspective, the experimental method is especially useful because it is expected that experimentation, especially when using random assignment to conditions and/or double-blind procedures, minimizes researcher biases and results in more accurate data. Within psychology, post-positivist views are assumed and not debated. Yet in education circles, criticism of post-positivism is not uncommon. Some maintain that bias can never be eliminated and trying to do so is futile and counter-productive. For example, Biesta $(2007 ; 2010)$ argues that post-positivist research is a managerial, top-down, non-democratic approach to evidencebased practice that is at odds with education as an inherently political and democratic practice where one's perspective should be valued, not minimized.

If positivism / post-positivism is on one end of the spectrum, then interpretivism is on the other (e.g., Taber, 2013). Interpretivism is focused on personal experiences and the importance of context. Furthermore, variations within individuals are influenced by context. Small sample sizes $(<10)$ are more common for interpretivist studies because of the value in delving into details of individual experience. In contrast, post-positivist studies tend to use larger samples to be able to make conclusions about populations rather than individuals. Taken together, the range of views about the subjectivity / objectivity of the data is often described as ontology (e.g., 
Cohen et al., 2011). In addition to ontology, these philosophies are linked to one's epistemology. Epistemology concerns the nature and origin of knowledge (e.g., Smeyers \& Smith, 2014). Epistemological views are very important for psychology and education, but the way that knowledge is viewed can be different both within and across these two fields.

Wellman and Gelman (1998) summarize how different viewpoints within psychology have constrained how new evidence about knowledge acquisition is generated and evaluated. Briefly, they outlined three psychological theories about knowledge acquisition: (1) different types of knowledge are stored and processed by specialized cognitive systems (modularity, e.g., Chomsky, 1988, Fodor, 1983); (2) knowledge is built through exposure and practice (information-processing, e.g., Chase \& Erikson, 1981; Chi, 1978) (3) knowledge is constructed through interacting and experimenting with the world (theory-theories, e.g., Gopnik \& Wellman, 1994; Piaget, 1953). If ontology and epistemology produce debates within psychology, then it follows that similar, if not more extensive debates, will occur between psychology and education. We have learned not to assume that what we view as good evidence of knowledge or learning will be shared universally.

Taken as a whole, educational research includes the spectrum of philosophies from interpretivism to post-positivism and a broad range of ontological and epistemological views (e.g., Taber, 2013). We have found that acknowledging our own ontological and epistemological positions early on and in informal conversations leads to more productive discussions and collaborations. Furthermore, our willingness to create a democratic approach to research design and to be open about other philosophical viewpoints have been important for our work in education. For us, this democratic approach has meant we avoid imposing our own perspective, and ways of doing things, and we are open to hearing teachers' perspectives about our design and measures. In one example of our wanting to take a more democratic approach, while remaining guided by scientific objectives, we modified our consent procedures when we included teachers as co-researchers. Treating them like research subjects did not feel 
appropriate when the studies were happening in their classrooms looking at their teaching practice. Asking them to sign a traditional consent form would imply that they are joining something that is above or outside of their agency (the notion of something 'being done to them'). Instead we created a memorandum of understanding, which was written in the firstperson plural and signed by our team and the teachers. The shift away from a consent form was indicative of an increased democratization of our research studies. This approach might not be appropriate for all projects, but we have learned to be flexible and open-minded about how we ensure ethical working, while not being overly stuck in a certain procedural way of doing things. As a result of being flexible and considering new ways of working, we find that we have increased buy-in from teachers and educators in our projects and that the studies progress more smoothly.

It is worth noting that recognition is increasing for post-positivist studies in education. In the UK, Goldacre (2013), echoing other authors from the USA like Kilpatrick (2001), argued that education should look to medicine to see the great value of the experimental method, and more specifically, the use of randomized control trials (RCTs). He presented cases where routine medical practices typically thought of as ethical were actually found to harm patients. He argued that without RCTs, the field of education might be susceptible to the continued use of harmful practices, or at the very least not improving practice in a systematic way. The UK government established the Education Endowment Fund (EEF) for the specific purpose of funding large scale RCT studies in education and showcasing the resulting findings. The EEF shares some goals with initiatives in other countries, like the What Works Clearinghouse of the Institute for Education Sciences, in the USA.

Goldacre (2013) and the work of the EEF have generated criticism from educational researchers and practitioners in the UK. One reason for these critiques is that many educators believe the parallel between medicine and education is flawed, on the basis that individual differences in learning and cognition are greater than individual differences in physiology, and 
so there cannot be a one-size-fits-all solution in education. Yet, the cutting edge of medical research does consider issues related to individual differences, and so does psychological research. For example, cancer treatments for individuals with the same type of cancer rarely have the same efficacy across patients. More recently, the Medical Research Council in the UK has suggested that some medical conditions, like developmental disorders, might not benefit from exclusive use of RCTs because of phenotypic heterogeneity (Craig, Dieppe, Macintyre, Michie, Nazareth, \& Petticrew, 2008; Moore et al., 2015). As such, the post-positivist position, and associated research methods, can be reconciled with attention to individual differences between children. At the same time, RCTs should be seen as only one among many suitable methods for establishing valid research findings in education.

“Good" student performance is important for teachers. Psychologists and educational practitioners do not always have a shared understanding of the desired outcomes in a study (e.g., Lendrum \& Humphrey, 2012). Without that shared understanding, unexpected complications can occur. We have seen some teachers expect certain levels of performance from children participating in an experiment, while we might want to "let the data speak". Put another way, teachers might see their job as supporting students to do well, while psychologists might see their job as revealing students' abilities (but not necessarily influencing them). In addition to teachers having a 'calling' to support children's learning, there are numerous incentives and punishments for educators and schools that do not meet performance targets.

This concern for student performance can show up in a number of ways. For example, one of us was part of a larger project that included a teacher-trainer who was fully committed to the experiment having seen the positive impact on their own students previously. Due to their enthusiasm, they were invited to help conduct some standardized psychology assessments. Despite being trained to follow the standardized protocols, and understanding the rationale for those protocols, they were unable to suppress coaching the participants towards the correct 
answer. This type of skill is vitally important for their success as a classroom teacher, yet a fundamental problem for psychology data collection.

While educators might see low levels of performance negatively, as if something is wrong with the measure, psychologists might use incorrect responses as a window into understanding the complexities of the cognitive mechanisms and how they change across ages. A good number of measures in psychology are designed for participants to make errors so that the influence of different conditions on performance can be investigated. Designing an evaluation where participants do not have a chance of getting everything right goes against many of the practices in classrooms. We have seen teachers try to prepare their students for our data collection by teaching their students about things they think we might evaluate in our study. In one case, a former teacher we employed gave teachers in the experimental group a copy of a standardized test that might be used as an outcome measure for an intervention. Of course, the teachers started practicing those items with their students before the scheduled data collection. Our team only knew that this training had occurred when a handful of children innocently mentioned that they had seen the items before. Trying to fully equip children for their evaluation was at odds with the fair test idea of good experimental design, while being totally consistent with educational practice. To head off any such misunderstandings, we are careful to explain why we pitch our measures at the level we do, and what the measures can tell us about cognitive development by being designed the way they are. We do not take for granted that evaluations of performance are used in the same way by both professions.

\section{Lesson III: Different Time Frames}

Flexible and responsive timings facilitate strong partnerships. One of the most frustrating challenges for us and the educators that we work with is how our two professions follow different timelines of working. Educators might take an idea, try it out, and continue to modify it while applying it to their practice because they want to see results immediately. This approach is often seen as good teaching. Psychologists might plan an investigation and see it 
through to the end, without tweaking things once the piloting phase is finished. This approach is often seen as good science. If the goal is to test a causal hypothesis, then the way psychologists approach their work is different from the way educators approach theirs. Different professional goals can produce these different timeframes.

We remind our teams that when they are hosted in schools, they should consider themselves guests and that they should be flexible to the timings and sensitive to changes that can happen at any moment in a school context. It can be frustrating for graduate students to prepare their materials and arrive at school only to be told the children are off on a field trip, but we try to let them know to expect such eventualities and to respond in good humor. Schools have fed back to us that they have appreciated such understanding on the part of the students.

We have reflected that there are different drivers for the pace of change when comparing psychology and education. Because the field of psychology generally sees its own work within the greater context of the complexity of cognitive mechanisms, psychologists tend to not want to apply findings in practice until they have strong evidence that theories are robust. The recent discussions about replication in psychology remind us that one study does not make a theory (more on this idea later). However, the nature of the teaching profession and the speed at which educational policy changes mean that educators seek to implement findings at a much faster pace. The drive to create better pedagogy means that educators always have their eyes open to the possible benefits of new ideas. A downside to this eagerness is they might adopt the latest fad that promises a quick fix, without solid evidence to support its effectiveness (e.g., Della \& Anderson, 2012; Hyatt, 2007; Kirschner, 2017)

This fast pace of change can affect participation as schools might find it difficult to commit to longer term projects. Furthermore, the appeal of how something new might have a positive impact on the school can make it difficult to recruit schools to participate if they know there is a chance that they are in a control group. We have had schools tell us that the only way they will participate is if they get the new program first. We have learned to avoid this outcome by 
involving schools in the design early and not only when we are looking for participants. In other words, when educators participate more fully at all stages of a research project, there is a better chance they grasp broader constraints and benefits and avoid common pitfalls of those who might be less research-literate. More buy-in, established by involving educators throughout the design process, can lead to greater investment in the importance of the project and the value of the chosen design. And importantly, this investment leads to an increased appreciation of how time consuming the research process can be, in general, so that the latest fads are seen with a more critical eye until they are more carefully vetted.

Educators apply psychology findings with or without us. It is natural for educators to want to use psychology and neuroscience findings to improve learning in classrooms. Unfortunately, in the time that it takes for innovations to be rigorously tested, creative marketers can start selling so-called brain-based products to schools that claim to make students smarter, without any empirical basis. For example, many educators in the UK still believe in the idea of every student having a learning style (kinesthetic, auditory, etc.; Dekker, Lee, Howard-Jones \& Jolles, 2012). Many not only buy in to this idea, but they actively use it to guide their teaching and to label students in their classrooms even though there is no empirical evidence to support this idea (Pashler, McDaniel, Rohrer, \& Bjork, 2009).

We have not found these issues of differing pace and goals easy to solve. However, one helpful tip when working with educators is simply to raise the issue as a point of discussion so that shared expectations can be established.

\section{Lesson IV: Different Semantics}

Frequent two-way conversations are needed to understand each other's language. It may seem an obvious point that scientific jargon is a barrier to wider communication outside of psychology. What is less obvious is how what seem like very straightforward terms can be used differently in different professions. For example, the term behavior means different things in the psychology community and in the UK teaching profession (the term may well be used differently 
again in other English-speaking communities). In psychology circles, the term behavior, refers to observable, outward manifestations of cognitive activity. In UK teaching circles, the term behavior often refers to acting out, specifically disruptive behavior that needs to be managed. Until one is aware of these different colloquial meanings for everyday words, there is the potential for talking at cross-purposes.

Likewise, we have encountered confusion over more specialized terminology. For example, some educators we have worked alongside do not understand the term cognition in terms of processes and mechanisms, as a psychologist would, but instead use the term to refer to the experience of thinking or the content of the thought itself. There are a good number of specialist terms that have different meanings (e.g., cognitive model, representation; see Devonshire \& Dommett, 2010 on the issue of shared vocabulary).

To address the culture gap in semantics, we have developed some strategies for conversations with educators. Experience has shown us that they usually want analogy and examples of what different cognitive constructs 'look like' in the classroom. For example, from our own studies with executive functions we have had various conversations with educators about inhibitory control. During those conversations, we have likened the concept of inhibitory control to a child's ability to ignore distractions in class. Of course, such simplifications do not capture the nuance of inhibitory control exactly as we might measure or conceptualize it in lab conditions. Instead, we have found it useful to build an understanding of the core concept, and to elaborate if necessary. This way of communicating can help one to focus on the essentials. Educators often want to know more than the definition of a construct from psychology, but what the implications could be for their practice and their understanding of student behavior. For example, when talking about executive functions, we have found that it can help to explain to educators that they underpin a child's ability to pay attention during class, and get work done instead of daydreaming. 
Frequent dialogue between various communities of practice is key to overcoming difficulties with semantics and jargon. The more opportunities there are to communicate, the more different groups can find common ground in their language, and misunderstandings can be more easily spotted and addressed (see Table 1). Conversations can take different shapes, such as online discussion platforms, workshops or collaborations on projects. Another benefit we have observed from building good communication with educators is that it fosters knowledge exchange and builds trust. Educators have often told us that their previous experiences have involved psychologists 'parachuting in' to schools to collect data and disappearing without any follow up or feedback. Building relationships where knowledge flow is genuinely bidirectional and sustained helps educators to feel included and valued in the psychology community. They appreciate a culture of mutual respect. The culture of trust that psychologists need to invest in when working with educators is analogous to how educators put in effort to build trusting relationships with the parents of children in their school: by modifying the language they use when talking to parents, being open to parents' views and being respectful of differing perspectives.

It is worth noting that building such relationships takes time and resource. One way that we have fostered general relationships with schools in the local area is through initiatives sponsored by our university, department or research centers. For example, putting on science festival activities, arranging free talks on topics of interest to educators and having administrators and assistant researchers help maintain the social media accounts. We focus on developing relationships through sustainable activities and not just linked to a specific project.

Communication needs to accommodate constraints. You could not blame a psychologist for thinking that teaching is mainly about learning. After all, learning is our bread and butter! However, it puts things in perspective to know that teachers in the UK have to demonstrate their ability to meet over 30 standards to become a qualified teacher, only one of which explicitly refers to understanding of how pupils learn (others include using relevant data to 
monitor progress, establishing rules and routines for the classroom, making a contribution to the wider life of the school, etc.; Department for Education, 2011). Likewise, teachers working in the USA and in other contexts might have many professional standards to uphold, in addition to their understanding of cognitive development. There is a common misperception on the part of psychologists that educators could easily test new ideas and take part in studies if they wanted. In our experience, educators' time is limited and divided amongst multiple concurrent priorities. Becoming aware of this constraint can be illuminating because it forces psychologists to think about how the evidence might be used: how does the suggested innovation fit with other constraints that educators face in the classroom? In our work on developing children's executive functions in the classroom by giving them more choices during the school day, collaborator educators pointed out that some schools have timetables mandated by school administrators, so they cannot follow children's interests outside of those structural constraints. This in turn influenced what kind of choices we focused our work on, so that they coincided with realities in the classroom (e.g., children choosing how long to spend on an activity might be less feasible than children choosing the subject of a project to complete).

High-quality, frequent communication can improve the uptake of the final result of work aimed at influencing pedagogy. Dialogue from the earliest stages is not uncommon in other fields like product design. Why has this practice eluded psychologists so much? For example, leaders in the television industry for children move very quickly from the initial conceptualization to consultations with focus groups and user testing, only to then return to the design process, and further elaborate their ideas. This seems intuitive when thinking of how industry operates; perhaps the scientific model simply has not been flexible enough in the past for us to consider how early dialogue with users might be incorporated. Whereas industry has a goal of making an appealing product, science has the goal of knowledge-building. Educators have reminded us that no matter how scientifically sound an intervention might be, if it does not respond to their needs, then it will not be adopted widely and any potential for impact at scale will be 
jeopardized. There appears to be a continuum from the most basic research with no foreseeable applications, to studies designed to have a direct effect on practice and/or policy (see Table 1). The more real-world impact prescribed by the objectives of the study, the more we find that we need to be working effectively across psychology and education.

In finding the best ways to support communication and innovation in this area, we have drawn on the knowledge and experiences of other psychologists who are interested in influencing policy and practice. The IDEAS Impact Framework ${ }^{\mathrm{TM}}$ was developed in response to the need for methodological innovation that supports applying findings to solve real-world problems (Center on the Developing Child at Harvard University, 2016). Co-creating interventions with stakeholders, testing a theory of change that links inputs and outcomes via explanatory mechanisms, and fast-cycle iteration are all features of the IDEAS approach. We have implemented these elements successfully in our own work. We encourage anyone considering adopting some of these methods to bear in mind the specific resources that are needed (see Figure 1). For example, building in time at the beginning of a grant for stakeholder engagement, paying for temporary cover for educators to collaborate with psychologists during their normal working hours, and adapting ethics protocols to account for the co-constructed nature of the educators' input all represent new ways of working relative to the traditional lab studies conducted by psychologists. We have benefited from institutional support in the form of seed funding, which has meant we can pay the costs associated with stakeholder engagement even before core grant funding has been secured.

Regular dialogue facilitates better evidence sharing. We have noted that psychologists sometimes believe that if only educators could spend more time acquainting themselves with the evidence about learning, then they would improve their practice. However, the practical implications of insights from psychology are not always clear. We have conducted a study investigating the role of play in developing children's executive functions and socialemotional competences (Gibson, Cornell and Gill, 2017) but, in our experience, ways of 
translating this into a pedagogical approach, action research project or school policy are rarely direct. As an example, one of our graduate students wanted to conduct a study that implemented improved recess opportunities, but found many local schools were not interested because they did not see a clear link from recess quality to student learning. A successful strategy to overcome this was to partner with a third-sector organization that supported improving play quality and already had national links to schools. They facilitated meetings between our team and the school principals (head teachers in the UK) who had adopted new recess models and those who were new to the idea. Opening up the conversation in this way improved things dramatically. The experienced principals shared how improving recess led to better concentration and behaviour during lessons. Benefits that were important to schools, which the psychologists could not have predicted based on the data, included reduced time waiting in line for lunch and fewer reports of playground injuries and incidents.

In the UK, there are initiatives that leverage online platforms to provide opportunities for educators to ask questions about learning and for psychologists to engage in an open dialogue with teachers (https://learning.imascientist.org.uk/). In the US, www.learningscientists.org is run by academics who are on a mission to share evidence in a bi-directional way between researchers and educators. In the UK, we were funded by the UK Economic and Social Research Council to organize a series of events known as The Educated Brain workshops, bringing together educators, psychologists and neuroscientists (www.neuroscience.cam.ac.uk/educatedbrain). In these events, we have talked about what tools and resources are needed by educators (and those who train them) so that they can authentically incorporate findings into classroom practices. For example, it is unrealistic to expect educators to simply inform themselves better of the most current findings, given institutional subscriptions to academic journals can be very expensive. Even if journal articles were freely accessible, as open access journals are increasingly widespread, educators simply do not have the time to read papers thoroughly, with a critical understanding of methods, and 
then to glean implications for their own schools or classrooms. More work is needed to literally translate findings for practitioners in short impactful reports aimed specifically at that audience. One reason translation might be lacking is the structural misalignment between the incentive structures in psychology and the access that educators have to research findings. In psychology, incentives revolve in large part around academic publications (e.g., UK Research Excellence Framework; USA tenure system), whereas educators might be more likely to access other forms of communication like blogs, podcasts and practitioner journals. These platforms need not be viewed as entirely mutually exclusive. For example, we and our graduate students have been invited to give talks to practitioner audiences, which are mirrored in published pieces in practitioner-facing journals (Baker, 2018; Özturk, Gibson, Howard \& Katsos, 2018; Paes \& Ellefson, 2018). These talks can be the subject of a blog for a general audience, which offers the opportunity to flesh out some theoretical connections, and practical implications, of empirical work. All of these accessible forms of publication, while not directly recognized in academic promotion, allow us to engage in a space for dialogue with practitioners. This can nourish the evolution of ideas, which in turn improves the overall quality of the research project. Communicating for different audiences, whether through podcasts, print or video, is a transferable skill that needs to be practiced in order to be improved. We are currently developing systems for our doctoral students and postdocs to contribute to this work. We encourage our teams to attend relevant communications training, as well as to add translational activities to their resumes.

Ensuring educators can engage with research requires an understanding of their professional context. Our Educated Brain workshops had funding to pay for substitute teaching, allowing delegates to be released from school to attend the workshops. They may be motivated to engage with psychologists as part of their own professional learning, but institutional pressures can sometimes be a barrier. These very practical considerations must be considered 
when planning projects so that sufficient resources are available to give access to all participants.

In summary, this section has explored four of the key lessons that we have learned by doing research projects in educational settings. We now turn to the second part of the paper, where we will each share how our research has changed as we have learned more about educational contexts and cultures.

\section{How Have We Changed?}

\section{Collaboration \& Shared Goal Setting Impact Research Designs}

As we identified at the start of the paper, we have independent streams of research that include executive functions. We describe below three case studies of how specific areas of our executive function research programs have been impacted by our collaborations with education. The case studies illustrate how our independent research questions and methods have been impacted by classroom constraints and observations, discussions of findings, and planning interventions. Collectively, we have learned firsthand the importance of engaging stakeholders at all stages of research (see Table 1) and we have seen how this early engagement has improved grant fundability, research designs, results and impact.

\section{Measuring Executive Functions in School Settings}

Ellefson's graduate research was in cognitive neuroscience - starting with event-related potential studies of infant memory and early language learning. In one study, unlike the previous studies, participants' responses indicated that they were searching for one *right* answer rather than being open to many. One key difference for the current participants, compared to the previous studies, was the onset of extensive testing that was happening in the United States (a precursor to the No Child Left Behind legislation). Discussions of these results led to a view that most theories of cognitive development ignore the role that schooling plays in thinking and reasoning skills. At the time, there was growing interest in collaborative research between 
cognitive science and education. As such, Ellefson designed a PhD dissertation using cognitive psychology methods that addressed questions that might be relevant to education. That design occurred before learning the lessons described in the first section and there were issues getting the work published because of her limited understanding of educational context and culture.

An inter-disciplinary post-doctoral post in cognition and education was where more educationally relevant research questions emerged and where Ellefson began investigating executive functions. Shortly after starting the first project, she administered some baseline arithmetic assessments for early primary school students ages 5 to 7 years that were designed by the co-PI from education. Some tasks were all addition or subtraction, where others were interleaved. The students had difficulty switching between addition and subtraction, and would perseverate on just one computation, especially on the interleaved parts. Ellefson knew a little about executive function research, so she started talking more to researchers about appropriate methods and tasks for designing a study that would look at switching abilities in arithmetic in young children. At the time, there was some good evidence to suggest that children have difficulty switching between non-arithmetic tasks, but no evidence to say if this would happen in arithmetic. There seemed to be a juxtaposition between this research and the practice of mixing up items in test - making it possible that this fragile new skill was even more difficult for young children during testing because they had to manage both the difficulty of the new skill and switching between computations. The first study of this idea (Ellefson, Shapiro, \& Chater, 2006) indicated that switching in arithmetic is not the same as switching in domain-general contexts.

The realization that existing experiments did not fully answer her educationally relevant research question has been repeated a number of times in various ways across Ellefson's research program. The first big set of tasks was harmonizing the methods used with young children and those used with adults to create tasks that are suitable for middle/older childhood. In Ellefson et al. (2006), E-prime® (Schneider, Eschman, \& Zuccolotto, 2002) was used to present switching tasks, but when trying to extend this finding to older children (Ellefson, 
Blagrove, \& Chater, 2017) it became obvious that having a finite number of laptops and places to plug in those laptops was constraining the data collection. As such, it was necessary to move beyond local stimulus presentation software. After an extensive search about ten years ago for online stimulus presentation programs, it became clear that none were suitable. As such, the Thinking Games Site was created as part of a wide-scale project to look at the longitudinal development of executive function skills in children attending schools in high-poverty communities that was funded by the Institute of Educational Sciences (U.S. Department of Education). The goal of the website was to run data collection more efficiently using school computer rooms, making any data collection less disruptive for schools and allowing for a way of comparing data collected in schools to those in the lab. Group administration could occur from about 8 years of age and up, with the possibility of doing one-to-one administration with the same tasks down to 6 years of age, depending on the difficulty of the task. Later on, the site was used for international studies of language and multilingualism with executive function skills (Antonio, Grohmann, Kambanaros, \& Katsos, 2016; Veenstra, Antoniou, Katsos, \& Kissine, 2018) as well as a cross-cultural inter-generational study of executive functions of children and their parents from the United Kingdom and Hong Kong (Ellefson, Ng, Wang \& Hughes, 2017).

To date, there have been thousands of participants in a number of studies who have completed tasks using this website. Data collection on this wide of a scale has led to other research questions that could not have been imagined at the start. One new interest that only big data can afford has been looking at validity, reliability and measurement invariance (Aldercotte, Thomas, Willis, Foster, Serpell, Parr \& Ellefson, 2013; Chu, Ellefson, Ng, Wang \& Hughes, 2018; Lai, Ellefson, Ng, Wang, \& Hughes, 2018; Xu, Ellefson \& Hughes, 2018) and other measures of reliability for computerized executive function tasks and to explore how socioeconomic status is linked to executive function development (Ellefson, Zachariou, $\mathrm{Ng}$, Wang, \& Hughes, 2018; Francis, Serpell, Parr, \& Ellefson, 2017; Penford, \& Ellefson, 2018). This work has led to additional questions about the measurement of executive functions skills 
across modalities, with a specific interest in whether computerized tasks measure the same skills as the table-top tasks used more often with young children or in standardized tests (discussed in Ellefson et al., 2017).

The Thinking Games site is still a work in progress. A lot of lessons have been learned about servers, fire walls and the lack of consistent internet signals in many schools (sufficient for school needs but sometimes problematic for computer data collection). The site had to be adapted so that the amount of time the site communicates with the server - to avoid dropping the task, while not overloading or storing too much data locally on the school computer. So much of the grant funding was spent on the participant experience that there was not much left to create a good interface for researchers. This improvement is needed if the site is to be shared across a wide group of researchers. Further, the site is limited to schools and international locations that can access the server (without extensive firewalls). As such, one next step would be to develop an app that can be used where internet signals are unreliable. And, with future funding, one arm of the site and/or app could be developed with teachersallowing for even wider data collection and utility. Once those improvements are implemented, new research questions will emerge that include a wider and more international set of participants from diverse backgrounds and this could lead to a better understanding of the universality of executive functions. None of these ideas would have been possible without doing psychology research in classrooms and having frank conversations with teachers about both the research and the way it is conducted.

\section{Executive Functions and Language Development}

Gibson's research falls at the intersection of speech-language pathology and developmental psychology. Its main emphasis is on understanding the influence of environmental and interactional contexts on children with atypical development (e.g. Autism Spectrum Disorder, Attention Deficit Hyperactivity Disorders, Turner Syndrome). Gibson's research program includes exploration of topics such as academic achievement, multilingual 
upbringing, play, mental health and peer relationships. Her previous work has shown that UK policy makers have an interest in research evidence, but that up-take is relatively low and unsystematic (Gibson, Kelvin, \& Goodyear, 2015). A core feature of her current research program is that it was designed with the intention to generate insights that would influence policy makers, clinicians and practitioners in the classroom.

To achieve such influence, a practitioner orientation has been adopted from the start. For example, one stream of work focuses on the role that executive function skills may play in mediating between early multilingual exposure and later social communication development for individuals with neurodevelopmental conditions. At the onset of this project Gibson's team systematically reviewed the available literature and found that, contrary to commonly held practitioner perceptions, there is no evidence that multilingualism is detrimental to the development of children who have neurodevelopmental disorders. The review identified several gaps and limitations in the existing evidence base and was published as a practitioner review in a respected journal that is reviewed by practitioners as well as academics (Uljarevic, Katsos, Hudry, \& Gibson, 2016).

Gibson's team disseminated the review findings in a number of ways. They held seminars and discussion days to which they invited specialist teachers and speech- language pathologists and wrote an easy-to-follow summary in a practitioner-focused magazine (Özturk et al., 2018). This dissemination and feedback sparked discussions that fed into the team conceptualization of executive functions in future work. For example, emotional control emerged as an important concept for educators working with individuals with Attention Deficit Hyperactivity Disorder, therefore, measures were added that investigated the role of so-called 'hot' executive function skills (i.e., those that require emotional regulation). This broadened the team's theoretical perspective from our more traditional initial approach of focusing on inhibition, flexibility and working memory (Sharma, Gibson \& Katsos, 2018). 
Practitioners welcomed the findings and Gibson's plans for more robust quantitative investigation focused on linguistic and psychological theory. However, she observed that to influence practice substantively, a greater qualitative understanding of patient/student experiences was required alongside practice-based research into how best to support multilingual families. Thus, practitioner engagement had both theoretical and methodological influences on subsequent grant proposals that were eventually funded by the UK Arts and Humanities Research Council and the UK autism charity Autistica. It is worth noting that sharing the initial psychology-based findings with stakeholders improved the fundability of the grant proposal. A better understanding of how to effectively apply executive function findings to the practical everyday needs of teachers meant the proposal was attractive to organizations that are unlikely to fund traditional experimental psychology work.

The research program is now 2 years in and exciting results are emerging. Gibson organized a conference colloquium "Language, cognition and services provision for multilingual children with neurodevelopmental disorders: multidisciplinary perspectives" to share preliminary findings (Gibson \& Katsos, 2018). The papers showcase preliminary research findings by academics, educators and clinicians involved in the project.

Crucially, the channels for influencing practice are being actively nurtured throughout the research process and did not end at the design and funding stage. This example demonstrates how improving dialogue, perspective-taking and shared goal setting between psychologists and educators can increase the feasibility and meaningfulness and impact of evidence (Lendrum \& Humphrey, 2012). The likelihood of up-take in a practitioner community is an important factor in whether an evidence-based innovation will be effective or worth the investment, regardless of whether there are convincing lab-based findings to support its efficacy. Having a dialogue from the earliest stages with stakeholders can contribute to the attractiveness of knowledge generated through a project (see Table 1). If educators perceive a need in their own practice, which the study responds to, then they might be more likely to adopt the findings. Implementing 
interventions faithfully is a well-known challenge in educational research (O'Donnell, 2008). In some cases, an educator may feel an intervention holds them back (e.g., if strict dosages are prescribed) instead of allowing them to give it their all, leading them to deviate from the experimental protocol. For this reason, psychologists who aim to develop interventions can benefit from stakeholder input at the stage of formulating hypotheses and choosing outcome measures (Farran, Meador, Christopher, Nesbitt, \& Bilbrey, 2017).

\section{Executive functions for Autonomous Classroom Learning}

Baker trained as a cognitive developmental scientist, before moving into the education domain and seeking to apply what we know about children's learning to how we teach in schools. Her team is currently working on a project that aims to improve young children's autonomy in early science classrooms by supporting their executive functions and engagement in learning. Baker's team has embraced the feedback loop between basic research and applied research (see Table 1), to build stronger theories with real-world relevance (Kittredge, Day, Blaskova \& Baker, 2017). Her approach is based on the IDEAS Impact Framework: developing a theory of change and consulting teachers about how these theoretical mechanisms may be relevant to their classroom practice (Center on the Developing Child at Harvard University, 2016). Below are some examples of how consulting both theory and practice at the same time has influenced her perspective on executive functions in the early childhood classroom context.

At the start of a project on executive functions in the science classroom, Baker's team hosted a series of four workshops for teachers where they introduced theoretical concepts (e.g. executive functions and child-led learning). First, they asked teachers to try research-informed approaches in their classrooms. Later, teachers and researchers reconvened and discussed the feasibility and meaningfulness of the approaches.

Using this dual approach of developing theory and practice in parallel stands is different from the more tradition approach of identifying mechanisms in the lab before 'translating' the findings into practice. Instead, Baker's team has worked with teachers as co-researchers to 
inform the project from the early stages. From a teacher perspective, feasibility is as important as the underlying hypothetical case for the program. As end users, teachers tend to want to know that a new program is likely to have benefits they can see in the classroom context where they work, before they are willing to make changes to their professional practices. By consulting end-users early, she now routinely asks questions about feasibility and meaningfulness for teachers before launching into lab experiments that test cognitive learning mechanisms.

In the ongoing project on executive functions and autonomy in early science learning, this approach produced a shift in how Baker conceptualized executive functions. Initially, she theorized that executive functions were a necessary set of cognitive skills for children to work independently. After engaging in regular dialogue with a group of teachers, she learned that the implication that children should become 'independent' and entirely self-driven was not desirable from the teachers' point of view. This focus on the child as a solitary unit seems to be a natural implication of the way that executive functions are theorized and studied in cognitive developmental research (i.e., as a capacity at the individual level to self-regulate). However, teachers encouraged her to shift the emphasis away from children working independently (alone) towards working independently as well as in groups and consulting the teacher as needed., Her team adopted the idea of autonomy instead of targeting independence as the primary outcome. Autonomy could encompass working alone and social interactions. This change in research focus illustrates how discussions with classroom teachers at the earliest stages influenced the way executive functions are conceptualized. As a result, the outcomes shifted slightly and more ways of assessing executive functions were included in the research design, rather than solely relying on the traditional lab-based measures of executive functions. Traditional lab-based measures only evaluate executive functions where the child is given a task to complete alone (e.g., Baker, Friedman \& Leslie, 2010). Baker now includes measures where children's executive functions were assessed in more real-world settings that involve 
social interactions, such as via teacher reports and classroom observations (Eberhart \& Baker, 2018a; 2018b).

The combination of theoretical work and teacher consultations affects how Baker conceptualizes and measures executive functions, and it also affects the procedures she has adopted in other aspects of the research design. For example, teachers questioned her team about how lab-based experimental psychology findings on how to support executive functions (e.g., through contingent scaffolding) can be applied in the classroom, when most lab studies involve an adult working one-to-one with a child (e.g., Lee, Baker \& Whitebread, 2018). In contrast, teachers usually work with children in small or large groups. This simple insight led her team to develop some experimental procedures that involved supporting children's autonomy with one researcher working with pairs of children instead of one child at a time (Kittredge, Kulkarni, Day \& Baker, 2018).

Baker's research team has deliberately consulted a range of teachers and schools to gauge generalizability of this new idea. Teachers all agreed that supporting autonomy in classroom learning was a worthwhile pursuit and something they wanted to improve, but they also raised concerns about how different types of learners may respond to the child-led approaches we were trying out. For example, they asked about children who cannot easily express themselves verbally, or children who find it difficult to regulate their emotions. These considerations have practical implications for the nature of the intended program, and they have theoretical implications for the types of moderators that would eventually be included in the statistical models that the team would develop (and the measurement tools used to assess those characteristics). As such, Baker's close collaboration with teachers informed a key addition to the hypothesized models, making them directly related to the day-to-day practice of teachers and able to inform the psychological theory in new ways.

The work on improving children's autonomous classroom learning by supporting executive functions aims to create impact not just by changing teaching practice, but also by influencing 
policy (see Table 1). Baker's team has worked with, and taken advice from, the UK Early Intervention Foundation (EIF), a clearinghouse of research evidence intended to inform national policy. The EIF publishes ratings on the strength of evidence for a program's positive causal impact on child outcomes (see https://guidebook.eif.org.uk/eif-evidence-standards). For example, the EIF guidance states that high-quality interventions do not use newly designed instruments for outcome measures. Instead they should rely on outcome measures with known validity and reliability in the population under investigation. Whereas one might use a novel test in small-scale lab-based studies, the ensuing data would not be seen as convincing in largerscale interventions that can inform policy decisions. This has been influential in Baker's project design insofar as it limits the outcomes that can be assessed.

Approaching the research in this way, at the intersection of cognitive development, education and policy, has entailed both challenges and opportunities for Baker and her team. First, it takes a long time to engage with so many different stakeholders, as opposed to just drawing up a research design amongst academic colleagues. Time must be explicitly built in to the research cycle, with the associated grant funding. Second, to genuinely engage with stakeholder views means being flexible and really listening and being prepared to adapt plans from what was originally conceived. This open-mindedness does not always come naturally to scientists. On the plus side, it is exceedingly refreshing to work with colleagues in different fields, whose professional experience enhances psychology. This type of approach can improve the impact of academic research in the long run, because the research is more likely to respond to real concerns of teachers and more likely to be taken seriously by policy makers. It has also contributed to the theoretical conceptualization of executive functions, in return, by raising questions of how these may vary by context and whether individual lab-based measures are sufficient to capture the way executive functions work in classrooms (Eberhart, Gains, \& Baker, 2018). 


\section{Conclusions and Future Directions}

It is our view that psychology research could be much more influential, valid and generalizable if it adopted more shared agenda-setting and co-creation of research designs that resonate with both education and psychology. The lessons we learned early on in our journey as psychologists in a Faculty of Education prepared us to more effectively communicate and involve educators in our studies.

For a long time, the ideal of a scientist has been someone who makes new discoveries, and the publication process has meant that novelty has been rewarded more than confirmation or disconfirmation of existing ideas (Nosek et al., 2015). More cohesion around shared goals between psychology and education makes it possible to build bodies of evidence around findings that garner replicability and robustness sufficiently to be taken seriously by policymakers. To this end, psychology has seen an increase in efforts for better transparency and multi-site independent replication (Braver, Thoemmes \& Rosenthal, 2014; Duncan, Engel, Claessens, \& Dowsett, 2014; Open Science Collaboration, 2015). More psychologists are adopting transparent practices, such as pre-registering intervention protocols before testing their effectiveness and disclosing methodological details like exclusions, which collectively enhance the quality of bodies of evidence emerging from the field (Eich, 2014) and the likelihood that research might influence policy.

For psychologists wishing to go beyond generating knowledge and see their findings make a difference, it can help to understand which types of evidence are most likely to have an impact on practice in the real world. Psychologists might tend to be interested in cognitive mechanisms surrounding learning and educators might tend to be interested in academic outcomes, standardized tests, progression or engagement in school. However, policy-makers tend to be convinced by outcomes that are even broader. It might be like the difference between asking whether the motor or suspension of a car is most responsible for its speed, compared to asking which parts of the motor contribute the most. The type of outcome, and therefore the 
level of analysis where measures are targeted, is a feature of the design that can be incorporated early.

Now that we have started designing our studies with educational impact and policy in mind, we have a better understanding of the rationale behind our methods, and our data and theories are more robust. We still conduct experiments regularly, but we do so with credible educator buy-in. However, we have learned not to assume that studies designed to inform the understanding of cognitive development will necessarily have a direct impact in educational practitioner and policy circles. This sentiment was argued by William James more than a century ago (James, 1899).

There is increasing pressure for psychologists to make clear links between their academic research and societal impact. For example, the high-stakes national research review in the UK (Research Excellence Framework) requires specific evidence for impact stemming from findings. In general, improving impact is a difficult task (Cai, Morris, Hwang, Hohensee, Robison, \& Hiebert, 2017), but we have learned that more interactions and opportunities for dialogue and shared goal setting increase our chances of achieving impact. This process takes time and resources (i.e. needs to be planned into grants) and it works best if it is done deliberately. Even in cases where there are clear applications of findings, there can be barriers to taking evidence from traditional lab-based studies straight into policy circles if these studies were not conducted with this objective in mind. Differences in goals are often accompanied by differences in world-view and differences in methods. Dialogue is necessary to make those bridges between psychology and education communities, throughout the research process, and not just at the end when there are findings to share. 


\section{References}

Aldercotte, A. ${ }^{\text {RA }}$, Thomas, $\mathrm{K}^{\mathrm{RA}}$, Willis, $\mathrm{T}^{\mathrm{RA}}$, Foster, A. ${ }^{\mathrm{RA}}$, Serpell, Z., Parr, T., \& Ellefson, M. (2013, May). Hot executive function in at-risk children: The validity of the Hungry Donkey task. Poster presented at the $25^{\text {th }}$ annual meeting of the Association for Psychological Science, Washington DC, USA.

Antonio, K., Grohmann, K. K., Kambanaros, M., \& Katsos, N. (2016). The effect of childhood bilectalism and multilingualism on executive control. Cognition, 149, 18-30. DOI: 10.1016/j.cognition.2015.12.002

Baker, S. (2018). Cognitive skills for active learning in the early years. Impact: Journal of the Chartered College of Teaching, 3. Retrieved from: https://impact.chartered.college/issue/issue-3-developing-effective-learners/

Baker, S. T., Friedman, O., \& Leslie, A. M. (2010). The opposites task: Using general rules to test cognitive flexibility in preschoolers. Journal of Cognition and Development, 11, 240254. DOI: $10.1080 / 15248371003699944$

Biesta, G. (2007). Why “what works” won’t work: Evidence-based practice and the democratic deficit in educational research. Educational Theory, 57, 1-22. Dol: 10.1111/j.17415446.2006.00241.x

Biesta, G. (2010). Good education in the age of measurement: Ethics, politics and democracy. Routledge: New York.

Braver, S. L., Thoemmes, F. J., \& Rosenthal, R. (2014). Continuously cumulating meta-analysis and replicability. Perspectives on Psychological Science, 9, 333-342. DOI:

\section{$10.1177 / 1745691614529796$}

Cai, J., Morris, A., Hwang, S., Hohensee, C., Robison, V., \& Hiebert, J. (2017). Improving the impact of educational research. Journal of Research in Mathematics Education, 48, 2-6.

Center on the Developing Child at Harvard University (2016). From best practices to breakthrough impacts: A science-based approach to building a more promising future for 
young children and families. Retrieved from

https://developingchild.harvard.edu/resources/from-best-practices-to-breakthroughimpacts/

Chase, W. G., \& Erikson, K. A. (1981). Skilled memory. In J. R. Anderson (Ed.), Cognitive skills and their acquisition. Hillsdale, $\mathrm{NJ}$ : Erlbaum.

Chi, M. T. H. (1978). Knowledge structure and memory development. In R. Siegler (Ed.) Children's thinking: What develops? (p.73-96). Hillsdale, NJ: Erlbaum.

Chomsky, N. (1988). Language and problems of knowledge. Cambridge, MA: MIT Press.

Chu, C. P. K., Ellefson, M. R., Ng, F. F., Wang, Q., \& Hughes, C. (2018, May). Understanding the complexities in assessing literacy across different cultures: an investigation of 9-to-16year-olds' English literacy skills in the United Kingdom and Hong Kong. Poster presented at the 48th Annual Meeting of the Jean Piaget Society, Amsterdam, The Netherlands.

Cohen, L., Manion, L. \& Morrison., K. (2011). Research methods in education (7th Ed.). Routledge: New York.

Coolican, H. (2014). Research methods and statistics in psychology (6th Ed.). Psychology Press: New York.

Craig, P., Dieppe, P., Macintyre, S., Michie, S., Nazareth, I., \& Petticrew, M. (2008). Developing and evaluating complex interventions: the new Medical Research Council guidance. British Medical Journal, 337, a1655. DoI: https://doi.org/10.1136/bmj.a1655

Della, S., \& Anderson, M. (Eds.) (2012). Neuroscience in education: The good, the bad, and the ugly. Oxford: Oxford University Press.

Dekker, S., Lee, N. C., Howard-Jones, P., \& Jolles, J. (2012). Neuromyths in education: Prevalence and predictors of misconceptions among teachers. Frontiers in Psychology, 3, 429. DOI: 10.3389/fpsyg.2012.00429 
Department for Education (2011). Teachers' standards. Guidance for school leaders, school staff and governing bodies. Retrieved from:

https://www.gov.uk/government/publications/teachers-standards

Denzin, N., \& Lincoln, Y. S. (Eds.) (2017). The Sage handbook of qualitative research (5th Ed.) Sage: London.

Devonshire, I. M., \& Dommett, E. J. (2010). Neuroscience, Viable applications for education? Neuroscientist, 16, 349-356. DOI: 10.1177/1073858410370900

Duncan, G. J., Engel, M., Claessens, A., \& Dowsett, C. J. (2014). Replication and robustness in developmental research. Developmental Psychology, 50, 2417-2425. DOI: 10.1037/a0037996

Eberhart, J., \& Baker, S.T. (2018a). The context and purpose of executive functions assessments: Beyond the tripartite model. Talk presented at the European Association for Research on Learning and Instruction: Neuroscience and Education Special Interest Group, London, UK.

Eberhart, J., \& Baker, S. T. (2018b). Honing protocols and practices for studying self-regulation in classrooms. Talk presented at the European Association for Research on Learning and Instruction: Metacognition Special Interest Group, Zurich, Switzerland.

Eberhart, J., Gains, H., \& Baker, S. T. (2018). Context matters in executive functions assessments: The role of child agency. Poster presented at the International Mind, Brain and Education Society Conference, Los Angeles, California, USA.

Eich, E. (2014). Business not as usual. Psychological Science, 25, 4-6. DOI: 10.1177/0956797613512465

Ellefson, M. R., Blagrove, E., \& Chater, N. (2017). Are all switches the same? Switching costs in arithmetic and non-academic domains during late childhood and early adolescence. Manuscript in revision.

Ellefson, M.R., Ng, F.F., Wang, Q., \& Hughes, C. (2017). Efficiency of executive function: A two- 
generation cross-cultural comparison of samples from Hong Kong and the United Kingdom.

Psychological Science, 28, 555-566. DOI: 10.1177/0956797616687812

Ellefson, M.R., Shapiro, L.R., \& Chater, N. (2006). Asymmetrical switch costs in children.

Cognitive Development, 21, 108-130. DOI:10.1016/J.COGDEV.2006.01.002

Ellefson, M.R., Zachariou, A., Ng, F.F., Wang, Q., \& Hughes, C. (2018). A cross-cultural comparison of socio-economic status, executive functions, and numeracy skills. Manuscript submitted for publication.

Farran, D. C., Meadon, D., Christopher, C., Nesbitt, K. T., \& Bilbrey, L. E. (2017). Data-driven improvement in prekindergarten classrooms: Report from a partnership in an urban district. Child Development, 88, 1466-1479. DOI: 10.1111/cdev.12906

Fodor, J. A. (1983). Modularity of the mind. Cambridge, MA: MIT Press.

Francis, G., Serpell, Z., Parr, T., \& Ellefson, M.R. (2017, July). Executive functions and academic achievement in a high-poverty sample. Poster presented at the 36th Annual Conference of the Cognitive Science Society, London, UK.

Gibson, J.L., Cornell, M. \& Gill, T. (2017). A Systematic Review of Research into the Impact of Loose Parts Play on Children's Cognitive, Social and Emotional Development. School Mental Health, 9, 295-309. DOI: 10.1007/s12310-017-9220-9

Gibson, J. L., \& Katsos, N. (2018). Language, Cognition and Services Provision for Multilingual Children with Neurodevelopmental Disorders. Retrieved from osf.io/g4rxh.

Gibson, J., Kelvin, R., \& Goodyer, I. (2015). A call for greater transparency in health policy development: Observations from an analysis of child and adolescent mental health policy. Evidence \& Policy: A Journal of Research, Debate and Practice, 11, 7-18. DOI: $10.1332 / 174426414 \times 13940168597987$

Goldacre, B. (2013). Building evidence into education. Department for Education: London. Retrieved from: https://www.gov.uk/government/news/building-evidence-into-education. 
Gopnik, A., \& Wellman, H. M. (1994). The theory theory. In L. A. Hirschfeld \& S. A. Gelman (Eds.). Domain specificity in cognition and culture. New York: Cambridge University Press. Hyatt, K. J. (2007). Brain gym: building stronger brains or wishful thinking? Remedial and Special Education, 28, 117-124. DOI: 10.1177/07419325070280020201

James, W. (1899). Talks to teachers on psychology: And to students on some of life's ideals. New York: Henry Holt and Company.

Kilpatrick, J. (2001). Where's the Evidence? Journal for Research in Mathematics Education, 32, 421-427. DOI: 10.2307/749702

Kirschner, P. (2017). Stop propagating the learning styles myth. Computers \& Education, 106, 166-171. DOI: 10.1016/j.compedu.2016.12.006

Kittredge, A., Day, N., Blaskova, L., \& Baker, S. T. (2017). Developing a theory of change with teachers as co-researchers: Child-led early science education. Talk presented at the American Educational Research Association Conference, San Antonio, Texas, USA.

Kittredge, A., Kulkarni, K., Day, N., \& Baker, S. T. (2018). Teaching to learn and learning to teach: Scaffolding supports children's self-regulated learning. Poster presented at the International Mind, Brain and Education Society Conference, Los Angeles, California, USA.

Lai, R.P.Y., Ellefson, M.R., Ng, F.F., Wang, Q., \& Hughes, C. (2018, May). Elucidating the Role of Executive Functioning and Metacognition on Academic Outcomes. Poster presented at the European Association for Research on Learning and Instruction (EARLI) SIG 22 conference, London, UK.

Lee, M. K., Baker, S. T., \& Whitebread, D. (2018). Culture-specific links between maternal executive function, parenting, and preschool children's executive function in South Korea. British Journal of Educational Psychology, 88, 216-235. Dol: 10.1111/bjep.12221 
Lendrum, A., \& Humphrey, N.H. (2012). The importance of studying the implementation of interventions in school settings. Oxford Review of Education, 38, 635-652. DOI: $10.1080 / 03054985.2012 .734800$

McCulloch, G. (2002). 'Disciplines contributing to education'? Educational studies and the disciplines. British Journal of Educational Studies, 50, 100-119. DOI: 10.1111/14678527.t01-1-00193

Moore, G. F., Audrey., S., Barker, M., Bond, L., Bonell, C., Hardeman. W., Moore, L., O'Cathian, A., Tinati, T., Wight, D., \& Baird, J. (2015). Process evaluation of complex interventions: Medical Research Council guidance. British Medical Journal, 350, h1258. DOI: 10.1136/bmj.h1258

Nosek, B. A., Alter, G., Banks, G. C., Borsboom, D., Bowman, S. D., Breckler, S. J., ... \& Contestabile, M. (2015). Promoting an open research culture. Science, 348, 1422-1425. DOI: $10.1126 /$ science.aab2374

O'Donnell, C.L. (2008). Defining, conceptualizing, and measuring fidelity of implementation and its relationship to outcomes in K-12 curriculum intervention research. Review of Educational Research, 78, 33-84. DOI: 10.3102/0034654307313793

Open Science Collaboration. (2015). Estimating the reproducibility of psychological science. Science, 349, aac4716. DOI: 10.1126/science.aac4716

Özturk, I. Ö., Gibson, J. L., Howard, K., \& Katsos, N. (2018). How does multilingualism affect the communication of children with neurodevelopmental disorders? Royal College of Speech \& Language Therapists Bulletin, 791, 26-28.

Paes, T., \& Ellefson, M. R.(2018). Pretend play and the development of children's language skills. Impact, 2, 60-63.

Pashler, H., McDaniel, M., Rohrer, D., \& Bjork, R. (2009). Learning styles: Concepts and evidence. Psychological Science in the Public Interest, 9, 105-119. DOI: 10.1111/j.15396053.2009.01038.x 
Penford, R. \& Ellefson, M. R. (2018). Arithmetic achievement: The effect of executive functioning, numerical acuity, and socioeconomic status. Manuscript submitted for publication.

Piaget, J. (1953). The origins of intelligence in the child. London: Routledge \& Kegan Paul.

Schneider, W., Eschman, A., \& Zuccolotto, A. (2002). E-Prime User's Guide. Pittsburgh, PA: Psychology Software Tools Inc.

Sharma, C., Gibson, J. L., \& Katsos, N. (2018). Links between 'hot' and 'cool' executive functions and Attention Deficit Disorder traits in multilingual, primary school-aged children. Manuscript in preparation.

Smeyers, P., \& Smith, R. (2014). Understanding education and educational research. Cambridge: Cambridge University Press.

Taber, K. S. (2013). Classroom-based Research and Evidence-based Practice: An introduction. (2nd Ed.). London: Sage.

Uljarevic, M., Katsos, N., Hudry, K., \& Gibson, J. (2016). Practitioner review: Multilingualism and childhood developmental disorders, an overview recent research and discussion of clinical implications. Journal of Child Psychology and Psychiatry, 57, 1205-1217. DOI: 10.1111/jcpp.12596

Veenstra, A., Antonio, K., Katsos, N, \& Kissine, M. (2018). Resisting attraction: Individual differences in executive control are associated with subject-verb agreement errors in production. Journal of Experimental Psychology: Learning, Memory, and Cognition, 44, 1242-1253. DOI: $10.1037 / x I m 0000516$

Wellman, H. M., \& Gelman, S. A. (1998). Knowledge acquisition in foundational domains. In W. Damon, D. Kuhn, \& R. S. Siegler (Eds.), Handbook of child psychology, Volume 2: Cognition, perception, and language (5th Ed., p. 523-573). Hoboken, NJ: Wiley.

Xu, C., Ellefson, M., \& Hughes, C. (2018, April). A cross-cultural analysis of the development of 
executive function during preadolescence. Poster presented to the biennial meeting of the Society for Research on Adolescence, Minneapolis, Minnesota, USA. 


\section{Table 1}

Example interactions at the intersection of cognitive development, education, and policy.

\begin{tabular}{|c|c|c|c|c|}
\hline & \multicolumn{2}{|c|}{ Generating Knowledge } & \multicolumn{2}{|l|}{ Creating Impact } \\
\hline & $\begin{array}{l}\text { Basic } \\
\text { Research }\end{array}$ & $\begin{array}{l}\text { Applied } \\
\text { Research }\end{array}$ & $\begin{array}{l}\text { Change } \\
\text { Practice }\end{array}$ & $\begin{array}{l}\text { Influence } \\
\text { Policy }\end{array}$ \\
\hline $\begin{array}{l}\text { Provide } \\
\text { Access }\end{array}$ & $\begin{array}{l}\text { Educators host } \\
\text { psychologists in } \\
\text { classrooms }\end{array}$ & $\begin{array}{l}\text { Networks of } \\
\text { educators } \\
\text { interested in } \\
\text { psychology and } \\
\text { sustained } \\
\text { collaboration }\end{array}$ & $\begin{array}{l}\text { Relationship with } \\
\text { local government, } \\
\text { local school } \\
\text { networks }{ }^{1} \text { and } \\
\text { administrators }\end{array}$ & $\begin{array}{l}\text { Building networks } \\
\text { over long periods } \\
\text { for two-way } \\
\text { dialogue and } \\
\text { understanding }\end{array}$ \\
\hline $\begin{array}{l}\text { Consult } \\
\text { and } \\
\text { Share } \\
\text { Ideas }\end{array}$ & $\begin{array}{l}\text { Psychologists } \\
\text { attend } \\
\text { conferences and } \\
\text { workshops aimed } \\
\text { at educators, and } \\
\text { vice versa }\end{array}$ & $\begin{array}{l}\text { Focus groups and } \\
\text { workshops } \\
\text { designed for } \\
\text { psychologists to } \\
\text { understand } \\
\text { educators' } \\
\text { professional } \\
\text { contexts }\end{array}$ & $\begin{array}{l}\text { Pairs of } \\
\text { educators } \\
\text { support each } \\
\text { other to feel } \\
\text { empowered to } \\
\text { make change on } \\
\text { entrenched } \\
\text { practices }\end{array}$ & $\begin{array}{l}\text { Exchanges and } \\
\text { short visits } \\
\text { between } \\
\text { government and } \\
\text { non-government } \\
\text { institutions }\end{array}$ \\
\hline $\begin{array}{l}\text { Strategic } \\
\text { Guidance } \\
\text { and } \\
\text { Priority } \\
\text { Setting }\end{array}$ & $\begin{array}{l}\text { Educators sit on } \\
\text { advisory boards } \\
\text { for psychology } \\
\text { grants or projects }\end{array}$ & $\begin{array}{l}\text { Expert educators } \\
\text { offer views on } \\
\text { feasibility and } \\
\text { meaningfulness of } \\
\text { psychology } \\
\text { projects }\end{array}$ & $\begin{array}{l}\text { Educators are co- } \\
\text { researchers } \\
\text { testing classroom } \\
\text { innovations in } \\
\text { iterative feedback } \\
\text { loops }\end{array}$ & $\begin{array}{l}\text { Ongoing } \\
\text { dialogue; high- } \\
\text { profile } \\
\text { appearances; } \\
\text { committee } \\
\text { membership }\end{array}$ \\
\hline
\end{tabular}

Note $-{ }^{1}$ Local school networks can include, for example, school districts (USA), local educational authorities (UK) or academy chains (UK) 


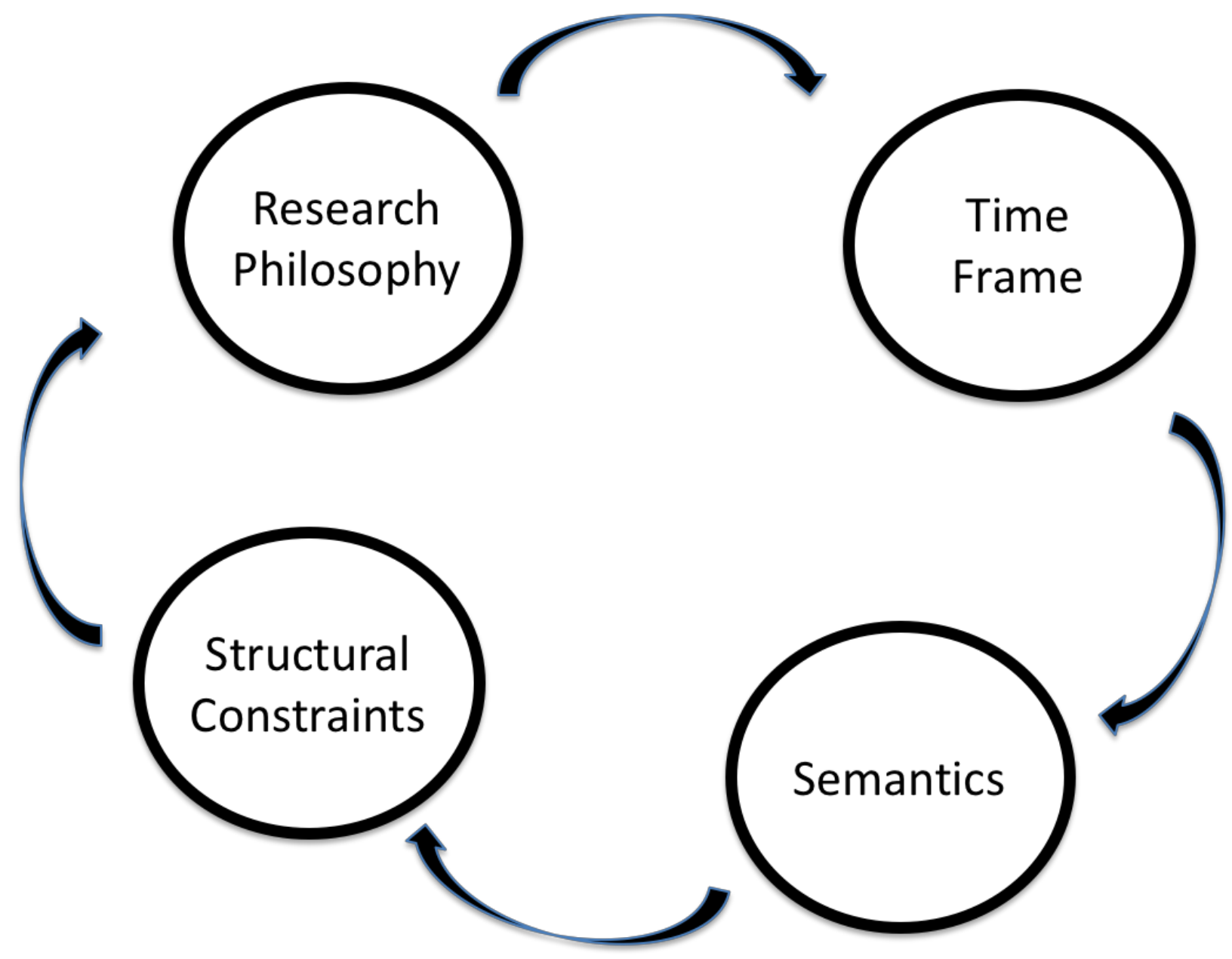

Figure 1. Considerations when designing a psychology research project at the intersection of cognitive development and education. 